Proceedings of the Edinburgh Mathematical Society (2003) 46, 35-44 (C)

DOI:10.1017/S0013091501000591 Printed in the United Kingdom

\title{
BORNOLOGICAL COUNTABLE ENLARGEMENTS
}

\author{
IAN TWEDDLE ${ }^{1}$ AND S. A. SAXON ${ }^{2}$ \\ ${ }^{1}$ Department of Mathematics, University of Strathclyde, Glasgow G1 1XH, UK \\ (i.tweddle@strath.ac.uk) \\ ${ }^{2}$ Department of Mathematics, University of Florida, \\ PO Box 118105, Gainesville, FL 32611-8105, USA (saxon@math.ufl.edu)
}

(Received 31 May 2001)

\begin{abstract}
We show that for a non-flat bornological space there is always a bornological countable enlargement; moreover, when the space is non-flat and ultrabornological the countable enlargement may be chosen to be both bornological and barrelled. It is also shown that countable enlargements for barrelled or bornological spaces are always Mackey topologies, and every quasibarrelled space that is not barrelled has a quasibarrelled countable enlargement.
\end{abstract}

Keywords: countable enlargement; bornological space; quasibarrelled space; Mackey topology

AMS 2000 Mathematics subject classification: Primary 46A08; 46A20

\section{Introduction}

We are concerned with the important class of bornological spaces, which may be defined as inductive limits of normed spaces and include all metrizable locally convex spaces. When the defining spaces are required to be Banach spaces we obtain the subclass of ultrabornological spaces; these are both bornological and barrelled and appear naturally in closed graph theorems. For properties of bornological and ultrabornological spaces see Chapter 6 of $[\mathbf{5}]$.

Let $(E, \mathcal{T})$ be a locally convex Hausdorff space with real or complex scalar field and dual $E^{\prime}$, and let $M$ be a subspace of the algebraic dual $E^{*}$ transverse to $E^{\prime}$ (i.e. $E^{\prime} \cap M=\{0\}$ ). The coarsest locally convex topology $\eta$ on $E$ finer than both $\mathcal{T}$ and $\sigma\left(E, E^{\prime}+M\right)$, denoted as $\sup \left\{\mathcal{T}, \sigma\left(E, E^{\prime}+M\right)\right\}$, is said to be an enlargement of $\mathcal{T}$. It has basic 0 -neighbourhoods of the form $U \cap A^{\circ}$, where $U$ is a basic $\mathcal{T}$-neighbourhood of 0 and $A$ is a finite subset of $M$. When $\eta$ is either barrelled or bornological, it is the Mackey topology $\tau\left(E, E^{\prime}+M\right)$, and in such a context the Mackey topology is sometimes taken as the definition of the enlargement corresponding to $M$, but the present definition seems more generally suitable (see [13]). If $M$ is finite- $\left(\aleph_{0^{-}}\right)$dimensional, then $\eta$ is a finite (countable) enlargement, abbreviated by FE (CE). An extension of the Banach-Mackey Theorem will show that there can be no disagreement as to what is an FE or CE of a barrelled or bornological space, since they all must be Mackey. That is to say, we will prove that if $(E, \mathcal{T})$ is 
barrelled or bornological and $\operatorname{dim}(M) \leqslant \aleph_{0}$, then the Mackey topology $\tau\left(E, E^{\prime}+M\right)$ is the only one finer than $\mathcal{T}$ for which the new dual becomes $E^{\prime}+M$.

In 1952 Dieudonné [3] proved that every finite-codimensional subspace of a barrelled (bornological) space is also barrelled (bornological) by essentially proving that FEs preserve the property of being barrelled (bornological). Obversely, preservation by finitecodimensional subspaces implies preservation by FEs: if $M$ is finite-dimensional, then $(E, \eta)$ is the direct sum of $M^{\perp}$ and a finite-dimensional subspace (see [5, 4.5.3]).

Proposition 1.1 (Dieudonné). Every FE of a barrelled (bornological) space is barrelled (bornological).

We note a related elementary fact.

Lemma 1.2. If $A$ is a barrel in a locally convex space $E$ such that $A \cap F$ is a 0neighbourhood in $F$ for some finite-codimensional subspace $F$, then $A$ is a 0-neighbourhood in $E$.

Proof. The closure $B$ of $A \cap F$ is a 0-neighbourhood in the closure $G$ of $F$, and $B \subset A$. Since $G$ is closed and finite-codimensional, any algebraic complement $H$ of $G$ is a topological complement, and $C=A \cap H$ is balanced, convex and absorbing in the finite-dimensional $H$, and therefore is a 0-neighbourhood in $H$. Thus $\frac{1}{2} B+\frac{1}{2} C$ is a 0-neighbourhood in $E$ that is contained in $A$.

The countable case is more complicated: every countable-codimensional subspace of a barrelled space is barrelled $[\mathbf{9}, \mathbf{1 8}]$, but if a barrelled space admits a CE, it admits one that is not barrelled [6]. On the other hand, countable-codimensional subspaces of bornological spaces need not be bornological [20], although countable-codimensional subspaces of ultrabornological spaces are always bornological $[\mathbf{1 9}]$. Of course there are bornological spaces that admit CEs with every $\mathrm{CE}$ bornological. For example, if $E$ is any infinitedimensional metrizable space, then every $\mathrm{CE}$ for $E$ is metrizable, hence bornological, and $E$ surely admits a CE: let $\left\{B_{n}\right\}_{n=1}^{\infty}$ be a pairwise disjoint partition of a denumerable linearly independent set; by multiplying the elements of $B_{n}$ by suitable non-zero scalars we may assume that each $B_{n}$ forms a null sequence in $E$; then take $f_{n} \in E^{*}$ such that $f_{n}\left(B_{m}\right)$ is $\{1\}$ for $n=m$ and is $\{0\}$ otherwise, and set $M=\operatorname{span}\left(\left\{f_{n}: n \in \mathbb{N}\right\}\right)$. In fact, if $E$ is either barrelled or bornological with $E^{\prime} \neq E^{*}$ (i.e. with $E$ non-flat in the terminology of [12]), then $E$ admits a CE. The barrelled case is proved in [6], and when $E$ is bornological it is non-flat if and only if there exists an infinite-dimensional bounded set, which allows us to proceed with null sequences $B_{n}$ as above.

The barrelled countable enlargement problem asks whether every non-flat barrelled space has a barrelled countable enlargement (BCE). It is known that BCEs exist for many important types of barrelled spaces (see $[\mathbf{6}, \mathbf{8}, \mathbf{1 0}-\mathbf{1 2}, \mathbf{1 6}]$ ); we note in particular for later application that every non-flat barrelled normed space has a BCE (see [11] and also [16]). In the present note we are concerned with the preservation of the bornological property under countable enlargement. In contrast to the failure of countable-codimensional subspaces of bornological spaces to be bornological in general, we find that every non-flat bornological space has a bornological $\mathrm{CE}$, from which we deduce that every non-barrelled 
quasibarrelled space has a quasibarrelled $\mathrm{CE}$. It was shown in $[\mathbf{1 6}]$ that a non-flat ultrabornological space always has a BCE; now we are able to extend this result to obtain a $\mathrm{CE}$ which is both barrelled and bornological. We begin with a consideration of conditions under which a CE is a Mackey topology and conclude with several examples to illustrate our results.

\section{Mackey topologies and enlargements}

Proposition 1.1 implies that every FE of a barrelled or bornological space is Mackey. We obtain the same result for CEs. However, some Mackey spaces have non-Mackey FEs $[\mathbf{5}, 4.5 .2(\mathrm{ii})]$. Let us say that the enlargement $\eta$ is Mackey inducing (MI) if it has the property that balanced convex $\sigma\left(E^{\prime}+M, E\right)$-compact sets contain only finite-dimensional subsets transverse to $E^{\prime}$ (a set $A \subset E^{*}$ is transverse to $E^{\prime}$ if $E^{\prime} \cap \operatorname{span}(A)=\{0\}$ ). Thus $\eta$ is $\mathrm{MI}$ if and only if each balanced convex $\sigma\left(E^{\prime}+M, E\right)$-compact set is contained in $E^{\prime}+N$ for some finite-dimensional subspace $N$ of $M$.

Theorem 2.1. An enlargement $\eta=\sup \left\{\mathcal{T}, \sigma\left(E, E^{\prime}+M\right)\right\}$ of a space $(E, \mathcal{T})$ coincides with the Mackey topology $\tau\left(E, E^{\prime}+M\right)$ if and only if $\eta$ is $M I$ and $L^{\perp}$ is a Mackey subspace of $(E, \mathcal{T})$ for each finite subset $L$ of $M$.

\section{Proof.}

Necessity. Suppose $\eta$ is Mackey and $A$ is a balanced convex $\sigma\left(E^{\prime}+M, E\right)$-compact set. Then $A^{\circ}$ is a 0 -neighbourhood in $(E, \eta)$, which means that $U \cap L^{\circ} \subset A^{\circ}$ for some $\mathcal{T}$ neighbourhood $U$ of 0 and some finite subset $L$ of $M$. Any $f \in A$ is numerically bounded by 1 on $U \cap L^{\perp}$, hence has a $\mathcal{T}$-continuous restriction to $L^{\perp}$, and thus $f=g+h$ for some $g \in E^{\prime}$ and some $h \in \operatorname{span}(L)$, so that $A \subset E^{\prime}+\operatorname{span}(L)$. Therefore, $\eta$ is MI. Furthermore, if $L$ is any finite subset of $M$, then $\left(L^{\perp}, \eta\right)$ has a topological complement in $(E, \eta)$ and thus is a Mackey space. Now if a balanced convex $C \subset\left(L^{\perp}, \mathcal{T}\right)^{\prime}$ is $\sigma\left(\left(L^{\perp}, \mathcal{T}\right)^{\prime}, L^{\perp}\right)$ compact, then it is $\sigma\left(\left(L^{\perp}, \eta\right)^{\prime}, L^{\perp}\right)$-compact, so that its polar $B$ in $L^{\perp}$ is a barrel in $\left(L^{\perp}, \mathcal{T}\right)$ and a 0 -neighbourhood in $\left(L^{\perp}, \eta\right)$. By definition of $\eta$, then, $B$ intersects some finite-codimensional subspace of $\left(L^{\perp}, \mathcal{T}\right)$ in a 0 -neighbourhood, so that by Lemma $1.2 B$ is a 0 -neighbourhood in $\left(L^{\perp}, \mathcal{T}\right)$. This proves that $\left(L^{\perp}, \mathcal{T}\right)$ is Mackey.

Sufficiency. Let $D$ be a balanced convex $\sigma\left(E^{\prime}+M, E\right)$-compact set with polar $D^{\circ}$ in $E$. Since $\eta$ is MI, there is some finite-dimensional subspace $L$ of $M$ such that $D \subset E^{\prime}+L$, and $\left(L^{\perp}, \mathcal{T}\right)$ is Mackey by hypothesis. The set of restrictions $\left.D\right|_{L^{\perp}}$ may be thought of as a subset of $E^{\prime}$ ( $L^{\perp}$ is $\mathcal{T}$-dense), which, with the topology induced by $\sigma\left(E^{\prime}, L^{\perp}\right)$, is a continuous image of $D$ and is therefore compact. Its polar in $L^{\perp}$ is $D^{\circ} \cap L^{\perp}$, a 0 neighbourhood in the Mackey space $\left(L^{\perp}, \mathcal{T}\right)$, and thus in $\left(L^{\perp}, \eta\right)$. Lemma 1.2 implies that $D^{\circ}$ is a 0 -neighbourhood in $(E, \eta)$.

If $A$ is a bounded balanced convex set in a space $E$ (i.e. a disc in $E$ ), we may give its span $E_{A}$ the norm which is the gauge of $A$. The closed unit ball in $E_{A}$ is just the algebraic closure of $A$. If $E_{A}$ is a Banach space, then $A$ is a Banach disc. The BanachMackey Theorem states that if $A$ is a Banach disc in $\left(E^{\prime}, \sigma\left(E^{\prime}, E\right)\right)$, then $A$ is $\beta\left(E^{\prime}, E\right)$ - 
bounded $[\mathbf{4}, \S 20.11(3)]$. We combine the proof with the result of $[\mathbf{9 , 1 8}]$ to obtain a useful extension.

Lemma 2.2. If $\eta=\sup \left\{\mathcal{T}, \sigma\left(E^{\prime}+M, E\right)\right\}$ is an FE or a $C E$ of a space $(E, \mathcal{T})$ and $D$ is a Banach disc in $\left(E^{\prime}+M, \sigma\left(E^{\prime}+M, E\right)\right)$, then $B=D \cap E^{\prime}$ is $\beta\left(E^{\prime}, E\right)$-bounded.

Proof. Since $M$ has countable dimension, $\left(E^{\prime}\right)_{B}$ is a countable-codimensional subspace of the Banach space $\left(E^{\prime}+M\right)_{D}$ and thus is barrelled with norm the gauge of $B$. (Barrelledness also follows from Satz 2 in [1].) Since $B$ is $\sigma\left(E^{\prime}, E\right)$-bounded, each member of $E$ may be considered a linear functional on $E_{B}^{\prime}$ which is bounded on the unit ball and thus is continuous. Consequently, any $\sigma\left(E, E^{\prime}\right)$-bounded set in $E$ becomes a pointwise bounded subset of the dual of the barrelled space $\left(E^{\prime}\right)_{B}$, hence equicontinuous, hence uniformly bounded on the unit ball, hence on $B$. It follows that $B$ is uniformly bounded on each $\sigma\left(E, E^{\prime}\right)$-bounded set; equivalently, $B$ is $\beta\left(E^{\prime}, E\right)$-bounded.

Tsirulnikov [14] defined a space $E$ to be dual locally quasicomplete (dlq) if the strong dual $\left(E^{\prime}, \beta\left(E^{\prime}, E\right)\right)$ is locally complete; equivalently, $E$ is dlq if every bounded balanced convex closed set in $\left(E^{\prime}, \beta\left(E^{\prime}, E\right)\right)$ is a Banach disc. Every quasibarrelled space is dlq; in fact, this is the weakest of the weak quasibarrelled conditions in [5, Chapter 8]. Since it is a duality-invariant property, one easily finds non-Mackey dlq spaces with CEs that are thus non-Mackey. On the positive side we greatly extend $[\mathbf{5}, 4.5 .8]$ from barrelled spaces to dlq spaces.

Theorem 2.3. Every $C E \eta=\sup \left(\mathcal{T}, \sigma\left(E, E^{\prime}+M\right)\right)$ of a dlq space $(E, \mathcal{T})$ is $M I$.

Proof. Any balanced convex $\sigma\left(E^{\prime}+M, E\right)$-compact set $C$ is the unit ball for a Banach space $X$. By Lemma 2.2 and the dlq hypothesis, $B=C \cap E^{\prime}$ must also be a Banach disc, so that it spans a closed subspace $Y$ of $X$. Now $\operatorname{dim}(X / Y)=\operatorname{dim}\left(\left(E^{\prime}+X\right) / E^{\prime}\right) \leqslant$ $\operatorname{dim}\left(\left(E^{\prime}+M\right) / E^{\prime}\right)=\aleph_{0}$ implies that the dimension of the Banach space $X / Y$ is finite. Consequently, $C \subset E^{\prime}+L$ for some finite-dimensional subspace $L$ of $M$.

Theorem 2.1 suggests two ways to construct non-Mackey CEs of Mackey spaces. The space $(c, \mathcal{T})$ of all convergent scalar sequences given the Mackey topology $\tau\left(c, \ell^{1}\right)$, where the bilinear form is that of the pairing $\left\langle\ell^{\infty}, \ell^{1}\right\rangle$, is a Mackey space that is dlq, but its dense hyperplane $c_{0}$ is not Mackey, so that any $\mathrm{CE}$ of $\mathcal{T}$ that makes $c_{0}$ a closed hyperplane of $c$ is a MICE that is not Mackey. On the other hand, if we take $E$ to be an $\aleph_{0}$-dimensional Mackey space with $\operatorname{dim}\left(E^{*} / E^{\prime}\right)=\aleph_{0}$, then the CE for which $E^{\prime}+M=E^{*}$ is not a MICE, for we can find a bounded linearly independent sequence in $M$ whose $\sigma\left(E^{*}, E\right)$ closed absolutely convex envelope must be $\sigma\left(E^{*}, E\right)$-compact, but it is not contained in $E^{\prime}+N$ for any finite-dimensional subspace $N$ of $M$.

However, Valdivia proved that every finite-codimensional subspace of a quasibarrelled space is quasibarrelled [17], and thus Mackey. Combining this with Theorems 2.1 and 2.3 we now have our desired result.

Theorem 2.4. Every CE of a quasibarrelled space is Mackey.

Corollary 2.5. Every CE of a barrelled or bornological space is Mackey. 
Most (partial) solutions to the BCE problem involve the Tweddle-Yeomans Criterion [16, Theorem 2] in some version (cf. [8]). The criterion and its quasibarrelled analogue apply to non-countable enlargements as well.

Theorem 2.6. An arbitrary enlargement $\eta=\sup \left(\mathcal{T}, \sigma\left(E, E^{\prime}+M\right)\right)$ of a (quasi)barrelled space $(E, \mathcal{T})$ is (quasi)barrelled if and only if every $\left(\beta\left(E^{\prime}+M, E\right)-\right) \sigma\left(E^{\prime}+M, E\right)$ bounded set is contained in $E^{\prime}+L$ for some finite-dimensional subspace $L$ of $M$.

\section{Proof.}

Necessity. Suppose $(E, \eta)$ is (quasi)barrelled. If the subset $A$ of $E^{\prime}+M$ is $\left(\beta\left(E^{\prime}+M, E\right)\right.$-) $\sigma\left(E^{\prime}+M, E\right)$-bounded, it is $\eta$-equicontinuous and $A^{\circ \circ}$ is $\sigma\left(E^{\prime}+M, E\right)$-compact. Since $\eta$ is Mackey, Theorem 2.1 ensures that $\eta$ is MI, so that $A \subset A^{\circ \circ}$ is contained in some $E^{\prime}+L$ as required.

Sufficiency. Suppose $A$ is a $\left(\beta\left(E^{\prime}+M, E\right)-\right) \sigma\left(E^{\prime}+M, E\right)$-bounded set. By hypothesis there is a finite-dimensional subspace $L$ of $M$ such that $A \subset E^{\prime}+L$. Now $A^{\circ}$ is a barrel in $(E, \eta)$ and $\left(L^{\perp}, \mathcal{T}\right)$ is (quasi)barrelled with $\left.A\right|_{L^{\perp}} \subset\left(L^{\perp}, \mathcal{T}\right)^{\prime}$, so $\left(\left.A\right|_{L^{\perp}}\right)^{\circ}=A^{\circ} \cap L^{\perp}$ is a 0 -neighbourhood in $\left(L^{\perp}, \mathcal{T}\right)$, hence in $\left(L^{\perp}, \eta\right)$. Therefore, $A^{\circ}$ is a 0 -neighbourhood in $(E, \eta)$ by Lemma 1.2 , and $\eta$ is (quasi)barrelled.

Corollary 2.7. If $(E, \mathcal{T})$ is any non-flat barrelled space, then the enlargement $\eta=$ $\sup \left(\mathcal{T}, \sigma\left(E, E^{*}\right)\right)$ is not Mackey.

Proof. If $\eta$ were Mackey it would coincide with the barrelled topology $\tau\left(E, E^{*}\right)$, and then by the theorem any $\sigma\left(E^{*}, E\right)$-bounded set would be contained in $E^{\prime}+L$ for some finite-dimensional subspace $L$ of any algebraic complement of $E^{\prime}$ in $E^{*}$. It follows easily from this, again by the theorem, that any $\mathrm{CE}$ of $\mathcal{T}$ would be a $\mathrm{BCE}$, which is false [6, Theorem 3].

Remark 2.8. This corollary shows in particular that Theorem 2.4 and Corollary 2.5 are no longer valid in general if $C E$ is replaced by arbitrary enlargement.

\section{Bornological and quasibarrelled CEs}

Our two main results on bornological spaces arise as corollaries to the following general theorem. Recall that the statement ' $F$ is dominated by a bornological space' means that there is a finer locally convex topology on $F$ under which it is bornological; $G$ denotes $F$ with this finer topology.

Theorem 3.1. Let $E$ be a bornological space and $F$ a non-zero subspace of $E$ dominated by a bornological space $G$. Suppose $\eta$ is a $C E$ of $E$ corresponding to $M \subset E^{*}$ such that for every subset $A$ of $E^{\prime}+M$ transverse to $E^{\prime}$ the set $\left.A\right|_{F}$ of restrictions is transverse to $G^{\prime}$. If the $C E \gamma$ of $G$ corresponding to $G^{\prime}+\left.M\right|_{G}$ is bornological, then so is the CE $\eta$.

Proof. Let $g \in M \backslash\{0\}$ and choose $h \in E^{\prime}$ such that $\left.h\right|_{F} \neq 0$. Then by applying the hypothesis to $A=\{g+h\}$ we deduce that $\left.g\right|_{F} \neq 0$. It follows from this that the restriction 
mapping $\left.g \mapsto g\right|_{F}$ is an algebraic isomorphism of $M$ onto $\left.M\right|_{F}$; thus, as required for $\gamma$ to be meaningful, $\left.M\right|_{G}$ is an $\aleph_{0}$-dimensional subspace of $G^{*}$ transverse to $G^{\prime}$.

Since $E$ is bornological it is the inductive limit of all the normed spaces $X_{\iota}(\iota \in I)$ that dominate a subspace of $E$. The $\mathrm{FE}$ or CE of $X_{\iota}$ corresponding to $X_{\iota}^{\prime}+\left.M\right|_{X_{\iota}}$ yields a metrizable space $Y_{\iota}$ that is thus bornological. Let $E_{\iota}$ denote $G+Y_{\iota}$ endowed with the finest locally convex topology for which the canonical injections of $H=(G, \gamma)$ and $Y_{\iota}$ are continuous. This topology, finer than that induced by $E$, is Hausdorff, and $E_{\iota}$ is bornological, being the inductive limit of two such spaces. Now $(E, \hat{\eta}):=\lim \left\{E_{\iota}: \iota \in I\right\}$ is also bornological and $\hat{\eta}$ is finer than $\eta$, itself Mackey by Theorem 2.4. Thus we need only show that each $f \in(E, \hat{\eta})^{\prime}$ is also in $(E, \eta)^{\prime}=E^{\prime}+M$ to show that $\eta=\hat{\eta}$ is bornological.

For any such $f$ its restriction $\left.f\right|_{E_{\iota}}$ is continuous, as are, then, $\left.f\right|_{H}$ and $\left.f\right|_{Y_{\iota}}$. Thus there exist two finite subsets $L_{1}$ and $L_{2}$ of $M$ and 0-neighbourhoods $U_{1}$ and $U_{2}$ in $G$ and $X_{\iota}$, respectively, such that

$$
\{f\}^{\circ} \supset\left(U_{1} \cap L_{1}^{\circ}\right)+\left(U_{2} \cap L_{2}^{\circ}\right) .
$$

Thus the restriction $u$ of $f$ to $\left(G+X_{\iota}\right) \cap\left(L_{1} \cup L_{2}\right)^{\perp}$ is continuous and has, by the Hahn-Banach Theorem, a continuous linear extension $u^{-}$to $\left(G+X_{\iota}, \underset{\rightarrow}{\rightarrow}\left\{G, X_{\iota}\right\}\right)$. Thus $\left.f\right|_{G+X_{\iota}}$ and $u^{-}$both extend $u$ from the above finite-codimensional subspace, and there exists $g \in \operatorname{span}\left(L_{1} \cup L_{2}\right)$ such that $\left.f\right|_{G+X_{\iota}}=u^{-}+\left.g\right|_{G+X_{\iota}}$. Now $g$ is independent of the given $\iota \in I$ since

(1) $\left.f\right|_{G}$ certainly is,

(2) $G \cap\left(L_{1} \cup L_{2}\right)^{\perp}$ is dense in $G$ by hypothesis,

(3) $\left.u^{-}\right|_{G} \in G^{\prime}$ is thus uniquely determined by $f$, hence so is $\left.g\right|_{G}$, and

(4) as shown above, each member of $M$ is determined by its restriction to $G$.

Hence $\left.(f-g)\right|_{X_{\iota}}=\left.u^{-}\right|_{X_{\iota}}$ is continuous for each $\iota \in I$, which means that $f-g \in E^{\prime}$ and $f \in E^{\prime}+M$.

Corollary 3.2. Every non-flat bornological space $E$ has a bornological $C E$.

Proof. Since $E$ is non-flat it has an infinite-dimensional subspace $F$ dominated by a normed space $G$ and such a $G$ has a CE: for example, we may apply the construction given in $\S 1$, which provides a $\mathrm{CE}$ for any infinite-dimensional metrizable space. Suppose that the CE corresponds to the $\aleph_{0}$-dimensional subspace $N$ of $G^{*}$ transverse to $G^{\prime}$. Extend each element of $N$ to an element of $E^{*}$ by setting it equal to 0 on some fixed algebraic complement of $F$ in $E$ and let $M$ be the set of these extensions. The theorem now applies with this $M$.

Corollary 3.3. Every non-flat inductive limit of normed barrelled spaces has a bornological BCE. In particular, every non-flat ultrabornological space has a bornological BCE.

Proof. Referring to the proof of the previous corollary, we may now take $F$ to be an infinite-dimensional barrelled normed space and choose its $\mathrm{CE}$ to be a BCE [11]. It is 
easily seen that $M$ now provides a CE which satisfies the conditions of both Theorem 2.6 and Theorem 3.1 (cf. the proof of Theorem 1 in $[\mathbf{1 6}]$ ).

Corollary 3.4. If a quasibarrelled space $(E, \mathcal{T})$ has an infinite-dimensional bounded set, then it has a quasibarrelled $C E \eta$.

Proof. The dual $E^{\prime}$ of $(E, \mathcal{T})$ is contained in the dual $E^{\times}$of $E$ with its (finer) associated bornological topology $\mathfrak{T}$. Since $\mathcal{T}$ and $\mathfrak{T}$ have the same bounded sets, $(E, \mathfrak{T})$ is non-flat, and there exists an $\aleph_{0}$-dimensional subspace $M$ of $E^{*}$ transverse to $E^{\times}$such that $\hat{\eta}=\sup \left\{\mathfrak{T}, \sigma\left(E, E^{\times}+M\right)\right\}$ is a bornological CE of $(E, \mathfrak{T})$. Thus $\hat{\eta}$ is quasibarrelled and must satisfy the appropriate criterion of Theorem 2.6, as must, then, the $\mathrm{CE} \eta=\sup \left\{\mathcal{T}, \sigma\left(E, E^{\prime}+M\right)\right\}$ for $(E, \mathcal{T})$.

We see from Corollary 3.4 that, if there is a quasibarrelled space which does not have a quasibarrelled $\mathrm{CE}$, then its bounded sets must be finite-dimensional, in which case the space would be barrelled and quasicomplete. Now, if the bounded sets of $(E, \mathcal{T})$ are finite-dimensional, then the $\sigma\left(E^{\prime}, E\right)$-bounded sets coincide with the $\beta\left(E^{\prime}, E\right)$-bounded sets; moreover, the bounded sets for any enlargement of $\mathcal{T}$ are just the same as the $\mathcal{T}$-bounded sets, so that barrelledness and quasibarrelledness remain equivalent for an arbitrary enlargement of $\mathcal{T}$. The remaining corollaries are now evident.

Corollary 3.5. Every quasibarrelled space which is not quasicomplete has a quasibarrelled CE.

Corollary 3.6. Every quasibarrelled space which is not barrelled has a quasibarrelled CE.

Corollary 3.7. If the BCE question has a positive answer in the special case where the space has only finite-dimensional bounded sets, then the quasibarrelled CE question always has a positive answer.

\section{Examples}

In the first example we describe a Mackey space whose dual has infinite codimension in its algebraic dual, so that CEs exist for it, but no CE can be Mackey since the second part of the condition in Theorem 2.1 always fails. Here and below $\omega$ denotes the product of a countably infinite collection of copies of the scalar field; $\varphi$ is the corresponding direct sum.

Example 4.1. Let $E=\bigoplus_{\lambda \in \Lambda} \varphi(\tau(\varphi, H))$, where $H$ is a dense hyperplane in $\omega$ and $\Lambda$ is an infinite set. Note that the topology of $E$ is $\tau\left(E, E^{\prime}\right)$ and its dual is $E^{\prime}=\prod_{\lambda \in \Lambda} H$. We show that $\tau\left(E, E^{\prime}\right)$ does not induce the Mackey topology on any dense hyperplane of $E$. Let $e$ span a supplement of $H$ in $\omega$.

Let $F$ be any dense hyperplane in $E$ and choose $x^{*} \in E^{*}$ having $F$ as null hyperplane. Now $x^{*}=\left(x_{\lambda}^{*}\right)$, where $x_{\lambda}^{*} \in \omega$, and for each $\lambda \in \Lambda$ there are unique $y_{\lambda} \in H$ and scalar $\alpha_{\lambda}$ such that $x_{\lambda}^{*}=y_{\lambda}+\alpha_{\lambda} e$. Moreover, $x^{*}=\left(y_{\lambda}\right)+\left(\alpha_{\lambda} e\right)$ and $E^{\prime}+\operatorname{span}\left(\left\{x^{*}\right\}\right)=E^{\prime}+$ $\operatorname{span}\left(\left\{\left(\alpha_{\lambda} e\right)\right\}\right)$. Clearly, not all the $\alpha_{\lambda}$ can be zero. 
We can find a sequence $\left\{x_{n}^{\prime}\right\}$ in $H$ such that $x_{n}^{\prime} \rightarrow e$ under $\sigma(\omega, \varphi)$. Let $B$ be the $\sigma(\omega, \varphi)$-closed absolutely convex envelope of $\left\{x_{n}^{\prime}: n \in \mathbb{N}\right\}$, which is $\sigma(\omega, \varphi)$-compact, and put

$$
A=\left\{\left(\alpha_{\lambda} x\right): x \in B\right\} .
$$

Then $A \subseteq E^{\prime}+\operatorname{span}\left(\left\{\left(\alpha_{\lambda} e\right)\right\}\right)$, for if $x \in B$ we have $x=x_{1}+\alpha e$ for some $x_{1} \in$ $H$ and some scalar $\alpha$, from which we get $\left(\alpha_{\lambda} x\right)=\left(\alpha_{\lambda} x_{1}\right)+\alpha\left(\alpha_{\lambda} e\right)$. Moreover, $A$ is $\sigma\left(E^{\prime}+\operatorname{span}\left(\left\{\left(\alpha_{\lambda} e\right)\right\}\right), E\right)$-compact and absolutely convex. This follows from the fact that the mapping $t: \omega \rightarrow \prod_{\lambda \in \Lambda} \omega$ defined by $t(x)=\left(\alpha_{\lambda} x\right)$ is continuous and linear.

By the weak continuity of the restriction map, the set $\left.A\right|_{F}$ of restrictions of members of $A$ to $F$ is $\sigma\left(F^{\prime}, F\right)$-compact (note that $\sigma\left(E, E^{\prime}+\operatorname{span}\left(\left\{x^{*}\right\}\right)\right.$ ) induces $\sigma\left(F, F^{\prime}\right)$ on $F$ ). Then, if $\tau\left(E, E^{\prime}\right)$ induces on $F$ its Mackey topology, $\left.A\right|_{F}$ is necessarily equicontinuous on $F$; the denseness of $F$ in $E$ then implies that $A \cap E^{\prime}$ is equicontinuous on $E$, so that its $\sigma\left(E^{\prime}, E\right)$-closure would be $\sigma\left(E^{\prime}, E\right)$-compact. This is false since $\left(\alpha_{\lambda} x_{n}^{\prime}\right) \in A \cap E^{\prime}$ for all $n \in \mathbb{N}$.

We sketch the details of another example of this type.

Example 4.2. Let $E$ be a real or complex vector space of dimension $\aleph_{1}$, let $B$ be a basis in $E$ and let $E^{\prime}$ be the subspace of $E^{*}$ consisting of those linear forms which vanish on all but countably many elements of $B$; in the terminology of $[\mathbf{1}], E^{\prime}$ is the $\aleph_{0}$-dual of $E$ with respect to the basis $B$. Then $\tau\left(E, E^{\prime}\right)$ does not induce the Mackey topology on any dense hyperplane of $E$. This may be established by showing that any $f \in E^{*} \backslash E^{\prime}$ is the limit under $\sigma\left(E^{*}, E\right)$ of a $\sigma\left(E^{\prime}, E\right)$-bounded net indexed by the countable ordinals, whose $\sigma\left(E^{*}, E^{\prime}\right)$-closed absolutely convex envelope $A_{f}$ is contained in $E^{\prime}+\operatorname{span}(\{f\})$. The null hyperplane $F$ of $f$ cannot then be a Mackey space in the induced topology since $\left.A_{f}\right|_{F}$ is $\sigma\left(F^{\prime}, F\right)$-compact and absolutely convex but not equicontinuous.

According to Corollary 3.3 any non-flat ultrabornological space has a bornological BCE. The next example shows that such an enlargement need not be ultrabornological.

Example 4.3. From [15] or [21] (see also [5, 6.3.11, 6.3.12]) there exists a dense hyperplane $H$ of $\omega$ which is not ultrabornological. Let $E$ be an infinite-dimensional Banach space; it contains a dense barrelled subspace $F$ with codimension $\mathfrak{c}=\operatorname{dim}(H)$ (see, for example, [6, p. 107, Corollary 2]). Let $G$ be an algebraic complement of $F$ in $E$. Define $M \subset E^{*}$ such that $M^{\perp}=F$ and $\left(G, \sigma\left(G,\left.M\right|_{G}\right)\right) \approx H$. Then $F$ is a closed subspace of $\left(E, \tau\left(E, E^{\prime}+M\right)\right)$ and $E / F \approx H$, which is barrelled but not ultrabornological. Since barrelledness is a three-space property [7], the $\mathrm{CE} \tau\left(E, E^{\prime}+M\right)$ is barrelled and, since it is metrizable, is bornological, but is not ultrabornological since it has a quotient which is not.

Nevertheless, ultrabornological CEs can occur.

Example 4.4. Let $E$ be an ultrabornological space with a dense ultrabornological hyperplane $H$ (see $[\mathbf{2}]$ for an example of such a space). Let $f$ be any element of $E^{*}$ having $H$ as null hyperplane. Then $E$ is also ultrabornological under $\tau\left(E, E^{\prime}+\operatorname{span}\{f\}\right)$ since it is then the topological direct sum of $H$ and a one-dimensional subspace. It is 
now easy to see that

$$
\tau\left(\prod_{n=1}^{\infty} E, \bigoplus_{n=1}^{\infty}\left(E^{\prime}+\operatorname{span}\{f\}\right)\right)
$$

is a $\mathrm{CE}$ of

$$
\tau\left(\prod_{n=1}^{\infty} E, \bigoplus_{n=1}^{\infty} E^{\prime}\right)
$$

and $\prod_{n=1}^{\infty} E$ is ultrabornological under both topologies [5, 6.2.14].

Finally, we give an example of an ultrabornological space with a non-quasibarrelled CE. Since bornological spaces are always quasibarrelled this will show that a CE for an ultrabornological (and hence for a bornological) space need not be bornological (cf. Corollary 3.2).

Example 4.5. Let $(E, \mathcal{T})=\bigoplus_{n=1}^{\infty} E_{n}$ be the topological direct sum of infinite-dimensional Banach spaces $E_{n}$, and define $f_{n} \in E^{*}$ so as to vanish on $E_{m}$ for $m \neq n$, and to be discontinuous on $E_{n}$. The $(L B)$-space $E$ is ultrabornological, but its CE $\eta$ corresponding to $M=\operatorname{span}\left\{f_{n}: n \in \mathbb{N}\right\}$ is not quasibarrelled. Indeed, every $\eta$-bounded set $A$ is $\mathcal{T}$ bounded and hence contained in $\bigoplus_{n=1}^{k} E_{n}$ for some $k \in \mathbb{N}$, which means that $\left\{f_{n}: n \in \mathbb{N}\right\}$ is $\beta\left(E^{\prime}+M, E\right)$-bounded, denying the condition of Theorem 2.6.

Acknowledgements. Some of the work for this paper was done when S.A.S. visited the University of Strathclyde during part of April and May 2001. We are grateful to the EPSRC for support (GR/R37326/01) and to the University of Strathclyde for hospitality.

\section{References}

1. I. Amemiya And Y. Kōmura, Über nicht-vollständige Montelräume, Math. Annln 177 (1968), 273-277.

2. S. Dierolf and P. LurJe, Deux exemples concernant des espaces (ultra)bornologiques, C. R. Acad. Sci. Paris Sér. A 282 (1976), 1347-1350.

3. J. Dieudonné, Sur les propriétés de permanence de certains espaces vectoriels topologiques, Ann. Polon. Math. 25 (1952), 50-55.

4. G. KÖThE, Topologische lineare Räume, vol. I (Springer, 1960).

5. P. PÉrez Carreras And J. Bonet, Barrelled locally convex spaces (North-Holland, Amsterdam, 1987).

6. W. Robertson, I. Tweddle and F. E. Yeomans, On the stability of barrelled topologies, III, Bull. Austral. Math. Soc. 22 (1980), 99-112.

7. W. Roelcke And S. Dierolf, On the three-space problem for topological vector spaces, Collect. Math. 32 (1981), 3-25.

8. S. A. SAxon, Metrizable barrelled countable enlargements, Bull. Lond. Math. Soc. 31 (1999), 711-718.

9. S. SAXon And M. Levin, Every countable-codimensional subspace of a barrelled space is barrelled, Proc. Am. Math. Soc. 29 (1971), 91-96.

10. S. A. SAXon And W. J. Robertson, Dense barrelled subspaces of countable codimension, Proc. Am. Math. Soc. 107 (1989), 1021-1029.

11. S. A. SAXon And L. M. SÁnchez Ruiz, Barrelled countable enlargements and the bounding cardinal, J. Lond. Math. Soc. 53 (1996), 158-166. 
12. S. A. SAXon And I. TweddLe, The fit and flat components of barrelled spaces, Bull. Austral. Math. Soc. 51 (1995), 521-528.

13. S. A. Saxon, L. M. SÁnchez Ruiz And I. Tweddle, Countably enlarging weak barrelledness, Note Mat. 17 (1997), 217-233.

14. B. Tsirulnikov, On conservation of barrelledness properties in locally convex spaces, Bull. Soc. R. Sci. Liège 49 (1980), 5-20.

15. B. Tsirulnikov, Remarkable hyperplanes in locally convex spaces of dimension at most c, Can. Math. Bull. 24 (1981), 369-371.

16. I. TweddLe AND F. E. Yeomans, On the stability of barrelled topologies, II, Glasgow Math. J. 21 (1980), 91-95.

17. M. Valdivia, A hereditary property in locally convex spaces, Annls Inst. Fourier 21 (1971), 1-2.

18. M. VAldivia, Absolutely convex sets in barrelled spaces, Annls Inst. Fourier 21 (1971), $3-13$.

19. M. Valdivia, On final topologies, J. Reine Angew. Math. 251 (1971), 193-199.

20. M. VAldivia, Some examples on quasi-barrelled spaces, Annls Inst. Fourier 22 (1972), 21-26.

21. M. VAldivia, Sur certains hyperplanes qui ne sont pas ultra-bornologiques dans les espaces ultra-bornologiques, C. R. Acad. Sci. Paris Sér. A 284 (1977), 935-937. 